\section{A) Check for updates}

Cite this: Nanoscale, 2021, 13, 6965

\title{
Mechanism of magnetization reduction in iron oxide nanoparticles $\uparrow$
}

\author{
Tobias Köhler, (D)*a,b,c Artem Feoktystov, ${ }^{a}$ Oleg Petracic, ${ }^{b}$ Emmanuel Kentzinger, (D) ${ }^{b}$ \\ Tanvi Bhatnagar-Schöffmann, (DD b,c,d Mikhail Feygenson, ${ }^{\mathrm{e}}$ Nileena Nandakumaran, \\ Joachim Landers, ${ }^{f}$ Heiko Wende, (DD ${ }^{f}$ Antonio Cervellino, ${ }^{9}$ Ulrich Rücker, (DD ${ }^{b}$ \\ András Kovács, ${ }^{d}$ Rafal E. Dunin-Borkowski (D) ${ }^{d}$ and Thomas Brückel (DD ${ }^{b, c}$
}

\begin{abstract}
Iron oxide nanoparticles are presently considered as main work horses for various applications including targeted drug delivery and magnetic hyperthermia. Several questions remain unsolved regarding the effect of size onto their overall magnetic behavior. One aspect is the reduction of magnetization compared to bulk samples. A detailed understanding of the underlying mechanisms of this reduction could improve the particle performance in applications. Here we use a number of complementary experimental techniques including neutron scattering and synchrotron X-ray diffraction to arrive at a consistent conclusion. We confirm the observation from previous studies of a reduced saturation magnetization and argue that this reduction is mainly associated with the presence of antiphase boundaries, which are observed directly using high-resolution transmission electron microscopy and indirectly via an anisotropic peak broadening in X-ray diffraction patterns. Additionally small-angle neutron scattering with polarized neutrons revealed a small non-magnetic surface layer, that is, however, not sufficient to explain the observed loss in magnetization alone.
\end{abstract}

Received 4th December 2020 ,

Accepted 29th March 2021

DOI: $10.1039 / \mathrm{d} 0 \mathrm{nr} 08615 \mathrm{k}$

rsc.li/nanoscale mechanisms leading to the macroscopic properties of nanoparticles and in particular deviations from model expectations, such as the previously reported reduced magnetization compared to the (theoretical) bulk value remains difficult. ${ }^{19-31}$

Proposed models for the spatial distribution of the magnetization include the presence of a magnetic core-shell structure ${ }^{24}$ spin disorder around defects ${ }^{21}$ and a reduced magnetization in the core due to reversed moments and frustration. ${ }^{22}$ Small-angle neutron scattering experiments with polarized neutrons on spherical particles with diameters of $9.94 \mathrm{~nm}$ revealed a magnetically depleted surface layer of $0.3 \mathrm{~nm}$ with canted spins. ${ }^{26}$ However, it was also stated that the observed reduction in magnetization compared to the bulk values can not be achieved by this surface layer alone, but requires a uniform reduction of the magnetization in the nanoparticle core. Lattice shifts resulting in antiphase boundaries (APBs), i.e. a shift of the iron sublattice while keeping the oxygen lattice unchanged, have been proposed to explain the reduction in magnetization in the particle core. ${ }^{27,29}$ Mössbauer spectroscopy and magnetometry measurements performed on iron oxide nanoparticles with diameters of $15 \mathrm{~nm}$ suggested the lack of a magnetically dead layer. ${ }^{30}$ Iron oxide nanoparticles with overall size of $7.4 \mathrm{~nm}$ were shown to be homogeneously magnetized with no significant spin disorder at the surface. ${ }^{28}$ Common to all previous works is the limitation to few experimental techniques, allowing conclusions only on specific parts of the problem. 
In this study we combine neutron and synchrotron X-ray scattering techniques with magnetometry, transmission electron microscopy (TEM), elemental analysis and Mössbauer spectroscopy to obtain a complete as possible picture of the nanoparticle properties. We find that the nanoparticles possess a macroscopically reduced saturation magnetization, mostly due to the presence of antiphase boundaries as observed with high-resolution TEM (HR-TEM) and X-ray scattering and to a lesser extent due to a small magnetically depleted surface layer and cation vacancies.

\section{Experimental}

\subsection{Materials}

Commercially available iron oxide nanoparticles with nominal core diameters of $15 \mathrm{~nm}$ with oleic acid coating dispersed in toluene were purchased from Ocean NanoTech (San Diego, USA). These particles were synthesized in organic solvent at high temperature. ${ }^{32}$

\subsection{Transmission electron microscopy}

Transmission electron microscopy (TEM) and high-resolution TEM (HR-TEM) was carried out using a FEI Tecnai G2 F20 field emission transmission electron microscope in bright field mode, operated at $200 \mathrm{kV}$ at the Ernst Ruska-Centre (ER-C), Forschungszentrum Jülich $\mathrm{GmbH}^{33}$ The sample was prepared by drop coating a carbon layer supported by a $\mathrm{Cu}-$ grid. The contrast between the organic shell of the nanoparticles and the amorphous carbon substrate is relatively small, thus only the crystalline core of the particles is visible in the micrographs. Image simulations to verify the contrast were performed with the QSTEM software package. ${ }^{34}$

\subsection{Mössbauer spectroscopy}

Mössbauer spectra were recorded at $4.3 \mathrm{~K}$ in transmission geometry and constant acceleration mode using a ${ }^{57} \mathrm{Co}(\mathrm{Rh})$ source in a liquid helium bath cryostat. The particle dispersion was dried and the powder was mixed with chemically inert boron nitride to obtain a homogeneous sample of sufficient volume.

\subsection{X-ray scattering}

2.4.1. Total scattering. Synchrotron X-ray scattering experiments on dried nanoparticle powder were performed at the Material Science Beamline X04SA of the Swiss Light Source at the Paul Scherrer Institut (Villigen, Switzerland). ${ }^{35}$ After normalization and correction of the obtained powder pattern the pair distribution function (PDF) was calculated with PDFgetX3. ${ }^{36}$ Analysis of the PDF was carried out with PDFGUI. ${ }^{37}$

2.4.2. Small-angle X-ray scattering. Small-angle X-ray scattering (SAXS) experiments were performed at GALAXI (Gallium anode low-angle X-ray instrument) at Jülich Centre for Neutron Science (JCNS), Forschungszentrum Jülich $\mathrm{GmbH}^{38}$ The experiments were conducted at two detector distances (3535 and $835 \mathrm{~mm})$ to cover the entire $Q$-range $(4 \times$ $10^{-2}$ to $8 \mathrm{~nm}^{-1}$ ) available on the system. Ga Ko radiation with
$9243 \mathrm{eV}$ photon energy (wavelength: $\lambda=0.134 \mathrm{~nm}$ ), monochromatized by parabolic Montel-type optics was used. The beam was collimated by two 4-segment slits. The data were recorded on a Pilatus $1 \mathrm{M} 2 \mathrm{D}$ position sensitive detector. The isotropic detector images were radially averaged using the fit2D software. ${ }^{39}$ The data were corrected for the empty cell and solvent scattering and the scattering cross section per unit volume $\left(1 / V\left(\frac{\mathrm{d} \sigma}{\mathrm{d} \Omega}\right)\right)$ was obtained via the reference material FEP $1400 \AA$ A. Data analysis was performed with Jscatter. $^{40}$ The wavelength spread for GALAXI is $\Delta \lambda / \lambda \approx 3 \times$ $10^{-3}$ ( $\mathrm{Ga} \mathrm{K} \alpha_{1}$ and $\mathrm{Ga} \mathrm{K} \alpha_{2}$ not resolved) but is deemed small enough to not be considered in the fitting. ${ }^{41}$ For the experiment borosilicate glass capillaries from Hilgenberg $\mathrm{GmbH}$ with wall thickness of $0.05 \mathrm{~mm}$ and internal diameter of $2.0 \mathrm{~mm}$ (ref. 42) were filled with nanoparticle dispersions (0.02 and 0.002 vol\% of particles).

\subsection{Small-angle neutron scattering}

Small-angle neutron scattering with polarized neutrons (SANSPOL) was performed on KWS-1 operated by the Jülich Centre for Neutron Science at Heinz Maier-Leibnitz Zentrum (Garching, Germany). ${ }^{43,44}$ Neutrons with a wavelength of $4.9 \AA$ and polarization, $P \approx 90.5 \%$, were detected at two detector distances $(2 \mathrm{~m}$ and $8 \mathrm{~m}$ ) with initial beam polarization opposite to the magnetic field, spin down, and parallel to it, spin up (flipper efficiency $\varepsilon=0.998$ ). A diluted solution of spherical nanoparticles 0.38 vol.\% was measured at 5 contrast conditions. Contrast variation experiments allow to probe directly the nuclear core-shell structure of the nanoparticles and utilization of polarized neutrons allows the magnetic structure to be precisely determined from a simultaneous fit of the multiple datasets of magnetic-nuclear interference. Discrepancies, if present between the magnetic and nuclear core size, will allow conclusions on the presence and thickness of a magnetically dead or depleted layer at the particle surface. Different contrasts were achieved by mixing hydrogenated and deuterated toluene (h-toluene and d-toluene). The final solvent composition constituted $0,25,35,50$ and 80 vol.\% of d-toluene and the resulting scattering length densities (SLDs) with contrasts (difference in SLD between the nanoparticle core and solvent $\left(\Delta \mathrm{SLD}_{\text {core }}\right)$ and the organic shell and solvent $\left.\left(\Delta \mathrm{SLD}_{\text {shell }}\right)\right)$ are listed in Table 1. Quartz cuvettes (Hellma GmbH, Germany) of $1 \mathrm{~mm}$ thickness were filled with $300 \mu \mathrm{l}$ of the samples and put

Table 1 Contrasts in the samples prepared for SANS

\begin{tabular}{|c|c|c|c|}
\hline Deuteration (vol\%) & $\begin{array}{l}\text { SLD }_{\text {solvent }} \\
\left(10^{-6} \AA^{-2}\right)\end{array}$ & $\begin{array}{l}\Delta \mathrm{SLD}_{\text {core }} \\
\left(10^{-6} \AA^{-2}\right)\end{array}$ & $\begin{array}{l}\Delta \text { SLD }_{\text {shell }} \\
\left(10^{-6} \AA^{-2}\right)\end{array}$ \\
\hline 0.0 & 0.939 & 5.717 & 0.861 \\
\hline $24.8(1)$ & 2.111 & 4.545 & 2.033 \\
\hline $34.7(1)$ & 2.579 & 4.077 & 2.501 \\
\hline $49.6(1)$ & 3.283 & 3.373 & 3.205 \\
\hline $79.8(1)$ & 4.710 & 1.946 & 4.632 \\
\hline
\end{tabular}
$0.078 \times 10^{-6} \AA^{-2}, \mathrm{SLD}_{\text {core }}{ }^{\mathrm{a}}=6.656 \times 10^{-6} \AA^{-2}$. ${ }^{\mathrm{a}}$ Assuming $\gamma-\mathrm{Fe}_{2} \mathrm{O}_{3}$ 
in sample position where a horizontal magnetic field of $0.5 \mathrm{~T}$ was applied orthogonally to the incident neutron beam.

The data were corrected for the solvent background and empty cell scattering and normalized to absolute intensities by measuring secondary standard samples and the empty beam. For each polarization state $10^{\circ}$-sectors in detector plane parallel and perpendicular to the applied field direction were integrated with the aim of obtaining the purely nuclear scattering contribution and the magnetic-nuclear interference term, respectively. Data reduction and fitting was carried out with qtiKWS and qtiSAS. ${ }^{45}$

\subsection{Magnetometry}

Magnetometry was performed using a superconducting quantum interference device (SQUID) magnetometer (MPMS XL, Quantum Design). The nanoparticle dispersion was diluted in paraffin to $0.1 \mathrm{vol}$ \% of the original concentration such that interparticle interactions become negligible. Pieces of the solid nanoparticle-paraffin mixtures were extracted using ceramic tools to avoid contamination with ferromagnetic materials. Zero-field cooled (ZFC) curves were measured by first cooling the sample in zero magnetic field to $10 \mathrm{~K}$, then applying a magnetic field and subsequently recording the magnetization while heating to room temperature. This is followed by a field-cooled (FC) measurement being recorded upon cooling to $10 \mathrm{~K}$ with the same magnetic field applied. To confirm the absence of interparticle interactions aged zero field cooled (aZFC) measurements, that would be able to detect a collective superspin glass state, were performed by stopping the zero field cooling process at $120 \mathrm{~K}$ for $10^{4} \mathrm{~s}$ and then proceeding in the same way as for the regular ZFC curves. Magnetization vs. field curves were obtained by sweeping over a field range of -1 to $1 \mathrm{~T}$ for constant temperatures $(300$ and $10 \mathrm{~K})$. The low temperature curves were recorded after various cooling fields of $0,0.1$ and $1 \mathrm{~T}$. AC-susceptometry was performed using the AC-option of a Quantum Design MPMS-5S SQUID magnetometer with frequencies in the range of $0.1-1500 \mathrm{~Hz}$ at temperatures between $5 \mathrm{~K}$ and $300 \mathrm{~K}$.

\subsection{Inductively coupled plasma optical emission spectroscopy (ICP-OES)}

ICP-OES was carried out at the central Institute for Engineering, Electronics and Analytics (ZEA-3), Forschungszentrum Jülich $\mathrm{GmbH}$. Samples used for magnetometry measurements were extracted from the sample holder, microwave digested in $\mathrm{HNO}_{3}$ and $\mathrm{H}_{2} \mathrm{O}_{2}$ and then analyzed. Co, Fe, Ni, Gd, Cr and $\mathrm{Al}$ were considered for determination of the total weight present in the sample. Details are presented in ESI Table S2.†

\section{Results and discussion}

We discuss firstly the SAXS measurements, that provide the particle size and size distribution, being essential for the evaluation and interpretation of the subsequent part.

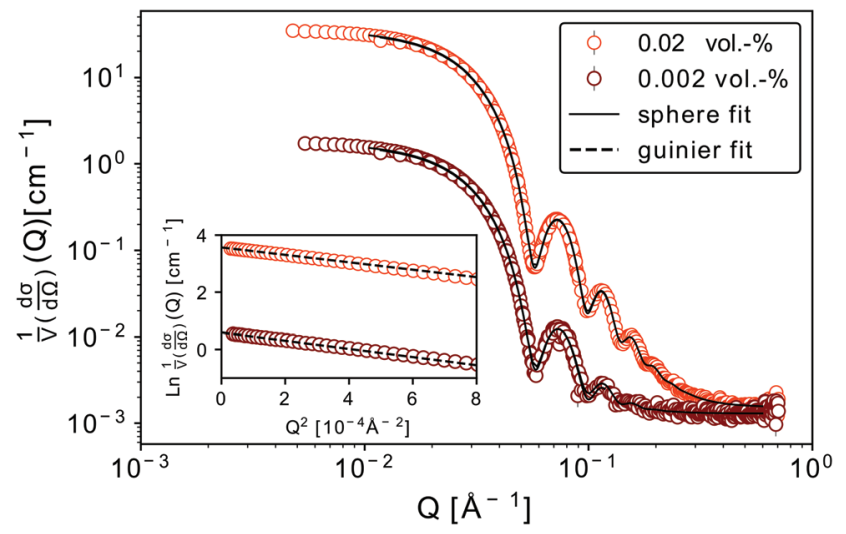

Fig. 1 SAXS scattering curves for two concentrations of nanoparticles dispersed in toluene. Clearly visible oscillations point to a well defined shape and small size distribution of the nanoparticles. The dashed lines in the inset correspond to a Guinier approximation of the low- $Q$ region $\left(\ln 1 / V(\mathrm{~d} \sigma / \mathrm{d} \Omega)\right.$ vs. $Q^{2}$ plot), the solid lines - to a solid sphere fit with particle radius and polydispersity as fitting parameters.

SAXS scattering curves (Fig. 1) measured from two samples with different particle concentrations (0.02 and 0.002 vol\%) were simultaneously fit to a model of polydisperse spheres with lognormal size distribution

$$
P\left(x, R_{0}, \sigma\right)=\frac{1}{\sigma x \sqrt{2 \pi}} \exp \left[-\frac{\left(\log \frac{x}{R_{0}}\right)^{2}}{2 \sigma^{2}}\right],
$$

which provided the distribution parameters $R_{0}=7.8(1) \mathrm{nm}$ and $\sigma=0.095(1)$.

For both concentrations of nanoparticles no interparticle effects were detected. A Guinier approximation of the low- $Q$ region in both cases shows no deviation from the linear behaviour in a $\ln I$ vs. $Q^{2}$ plot (see inset in Fig. 1), thus confirming that particles are non-interacting and similar in size. ${ }^{46}$ From a linear fit the radius of gyration, $R_{\mathrm{g}}$, of the nanoparticles can be extracted without assuming any model. $R_{\mathrm{g}}$ was found to be $6.3 \mathrm{~nm}$, which according to $R_{g}{ }^{2}=\frac{3}{5} R^{2}$, corresponds to a spherical particle radius of $7.9 \mathrm{~nm}$ in agreement with the particle size determined from the solid sphere fit. We have to emphasize that in the X-ray scattering, because of a vanishing contrast between surfactant shell and solvent, the signal originates mainly from iron oxide cores and contribution from the organic coating can be neglected. ${ }^{47}$

Magnetization curves, $M(H)$ and $M(T)$, normalized to the amount of iron present in the sample as determined by ICP-OES (ESI Table $\mathrm{S} 1 \dagger$ ), are displayed in Fig. 2. The maximum of the ZFC curve (Fig. 2(a)) lies at 172 K. For perfectly monodisperse nanoparticles with equal magnetic anisotropy energies this temperature marks the transition from a blocked to an unblocked state, i.e. the blocking temperature $T_{\mathrm{B}}$. For deviations from this ideal case the mean blocking temperature is found at slightly lower values. ${ }^{48,49}$ AC-susceptibility measurements on our samples (ESI Fig. S7†) suggest a mean blocking 

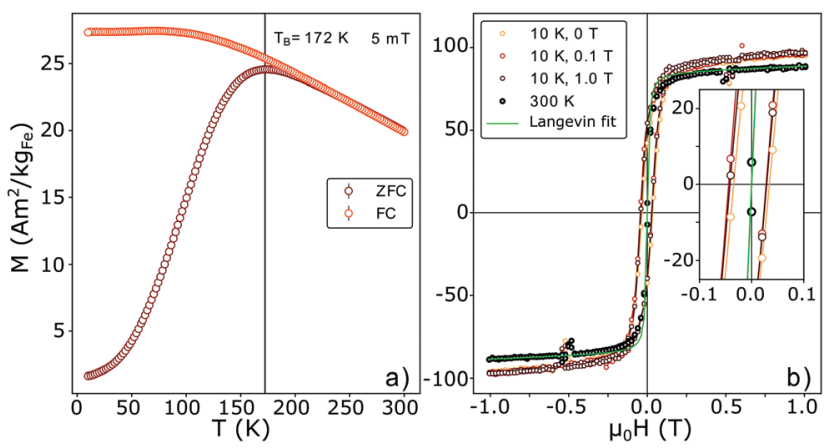

Fig. 2 (a) $M(T) Z F C$ and FC curves measured at $5 \mathrm{mT}$. The maximum of the ZFC curve and the splitting are in very close proximity, pointing to a small size distribution of the particles. The blocking temperature taken at the maximum of the ZFC curve yields $172 \mathrm{~K}$. (b) $M(H)$ curves measured at room-temperature (RT) and $10 \mathrm{~K}$ (with cooling fields of $0.0,0.1$ and $1.0 \mathrm{~T})$. The saturation magnetization $M_{\text {sat }}$ was normalized to the iron weight in the sample as determined via ICP-OES. A very small shift of the field cooled curves is visible. The green line corresponds to a Langevin fit (eqn (5)).

temperature of $160 \mathrm{~K}$. Together with the particle volume $V$, the measurement time $\tau_{\mathrm{m}}$ (taken as ca. $30 \mathrm{~s}$ ) and the elementary spin flip time $\tau_{0}$, which is typically on the order of $10^{-9} \mathrm{~s}$ for superparamagnetic systems in $\mathrm{ZFC} / \mathrm{FC}$ measurements, the magnetic anisotropy energy constant $K$ can be determined according to ${ }^{50}$

$$
K=\frac{k_{\mathrm{B}} T_{\mathrm{B}} \ln \tau_{\mathrm{m}} / \tau_{0}}{V}
$$

This results in a magnetic anisotropy energy of $27 \mathrm{~kJ} \mathrm{~m}^{-3}$, which is comparable to previously reported values for similar particles $^{51,52}$ and significantly larger than the maghemite magnetocrystalline anisotropy of $K=4.7 \mathrm{~kJ} \mathrm{~m}^{-3}$ obtained from measurements on single crystal films and powders. ${ }^{53}$ The close proximity of the splitting of the curves to the maximum of the ZFC curve points to a narrow particle size distribution ${ }^{50}$ which is consistent with the results obtained from SAXS experiments. The constant, i.e. not decreasing part of the FC curve for temperatures smaller than the ZFC peak temperature indicates the absence of a significant interparticle interaction $^{50}$ and the absence of an additional paramagnetic signal from impurity atoms or clusters dispersed between the particles. $^{54}$

A very small exchange bias field of $\mu_{0} H_{\mathrm{E}}=8 m T$ in the $0.1 \mathrm{~T}$ field cooled $M(H)$ curve was detected (inset in Fig. 2b). Exchange bias in iron oxide systems has previously been attributed to a ferro(ferri)magnetic/antiferromagnetic interface, e.g. the presence of wüstite $(\mathrm{FeO})$ in maghemite/magnetite $\left(\gamma-\mathrm{Fe}_{2} \mathrm{O}_{3} / \mathrm{Fe}_{3} \mathrm{O}_{4}\right)$ particles. ${ }^{5,56}$ On the other hand, it has been reported that antiphase boundaries produce exchange bias in thin films ${ }^{57}$ and according to Wetterskog et al. ${ }^{27}$ might also occur in nanoparticles. Such lattice translations modify the $\mathrm{Fe}_{\text {Oct. }}-\mathrm{O}-\mathrm{Fe}_{\text {Oct. }}$ bond angles across the APB from $90^{\circ}$ to $180^{\circ}$ (Fig. 3(e)). In agreement with the Goodenough-Kanamori
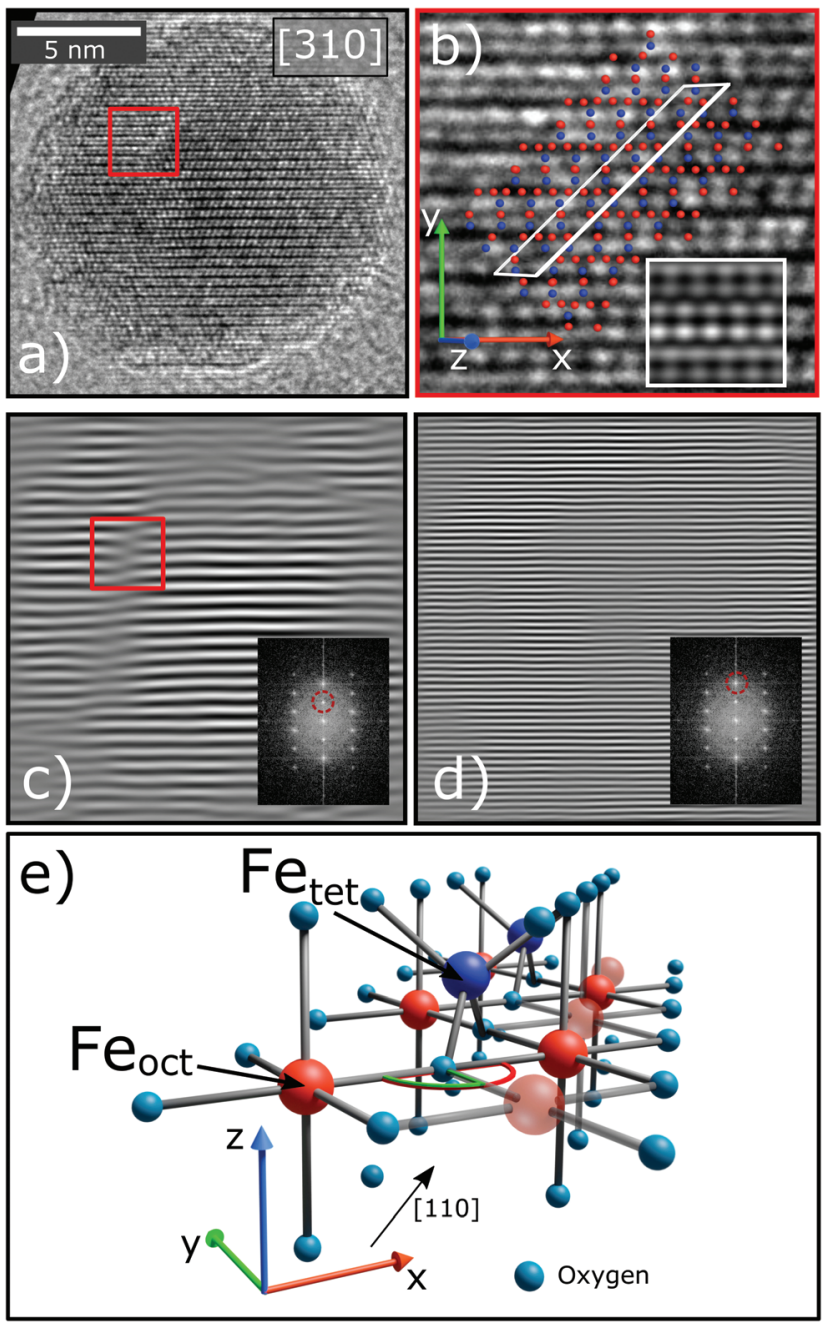

Fig. 3 (a) HR-TEM micrograph of an isolated nanoparticle viewed along [310]. A region with an APB is marked with the red square. (b) Marked region of (a) with a schematic of the crystal structure, the atom positions were verified by multislice TEM image simulations (inset). The lattice plane along which the translation occurs is indicated with the white rectangle. Red and blue dots represent iron atoms in octahedral and tetrahedral coordination, respectively. (c) The micrograph depicted in (a) after masking the 220 Bragg reflection (red circle in the inset) in the fast Fourier transform (FFT) and calculating the inverse FFT (IFFT). Now the lattice translation becomes clearly visible. (d) The IFFT after masking the 440 reflection, here the APB is not visible. (e) Schematic of the crystal structure viewed along [110] (black arrow) illustrating the bond angle change between octahedral atoms (red) from $90^{\circ}$ (green) to $180^{\circ}$ (red half-circle) through the iron sub-lattice shift.

rules $^{58,59}$ the superexchange interaction between iron cations at the APB was found to be antiferromagnetic via magneto-resistance measurements on $\mathrm{Fe}_{3} \mathrm{O}_{4}$ thin films. ${ }^{60}$ On the basis of Monte-Carlo simulations it was proposed that APBs are responsible for the drastic reduction of saturation magnetization $\left(M_{\text {sat }}\right)$ in iron oxide nanoparticles. ${ }^{29}$ Thus, checking for the existence of such defects in the studied samples is an important step towards answering the central question of the origin of the reduced $M_{\text {sat }}$. 
During the synthesis of monodisperse iron oxide nanoparticles via thermal decomposition of an iron-oleate complex $^{61}$ the particles pass through a stability field of the wüstite (FeO) phase that gets first oxidized to magnetite $\left(\mathrm{Fe}_{3} \mathrm{O}_{4}\right)$ and then to maghemite $\left(\gamma-\mathrm{Fe}_{2} \mathrm{O}_{3}\right)$. In this process it is common to introduce lattice shifts and antiphase boundaries (APBs). ${ }^{27}$ The presence of APBs in the samples of this study is directly confirmed by HR-TEM (Fig. 3). They can be more easily identified by masking the Bragg peak of the lattice planes that will be affected by this translation, in this case the220 reflection (Fig. 3(c)). The lattice shift is not affecting the oxygen sublattice (illustrated by masking the 440 peak in Fig. 3(d)) and does not change the net Fe coordination, leading to a very low formation energy. ${ }^{62}$ HR-TEM, while being very precise, can only provide statistically limited information on the particle structure. To confirm that APBs occur significantly often in the particles, larger ensembles have to be studied, which is provided by X-ray diffraction. This method probes the average crystal structure of the nanoparticles over a large sample volume and the presence of APBs can be deduced from selective peak broadening (Fig. 4).

A coherent structure size can be estimated from the FWHM of the strongest peaks using the Scherrer equation

$$
D_{h k l}=\frac{K \lambda}{B_{h k l} \cos \theta},
$$

where $D_{h k l}$ is the size of the investigated crystallite in the direction perpendicular to the set of planes with the Miller indices $h k l . K$ is a factor accounting for the crystallite shape, $\lambda$ is the X-ray wavelength, $B_{h k l}$ is the full-width at half-maximum (FWHM) of the corresponding Bragg peak in radians and $\theta$ is the scattering angle. ${ }^{63,64}$ The value of $K$ is usually taken as 0.94 for spherical particles with cubic unit cells. ${ }^{65} B_{h k l}$ for the peaks were extracted from Voigt profiles fitted to the experimental data and were then corrected for instrumental peak broaden-

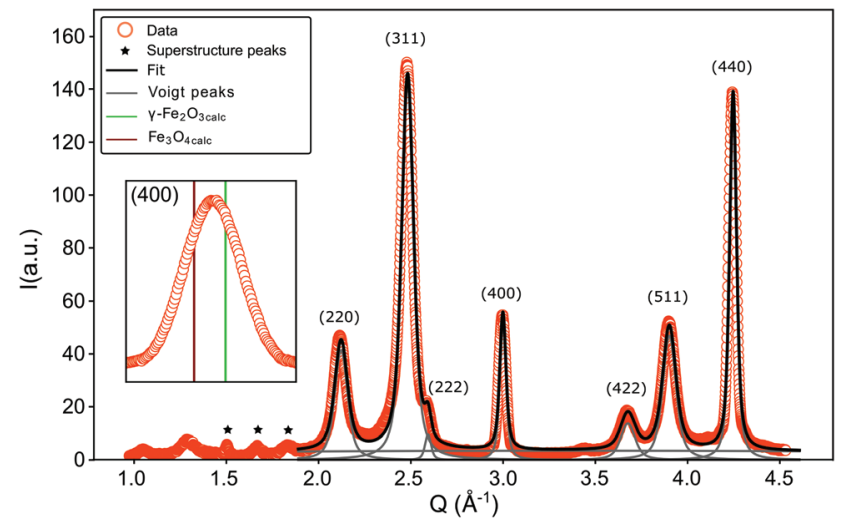

Fig. 4 X-ray scattering spectrum of the nanoparticle sample. FWHM of the strongest peaks were extracted from Voigt profiles. The inset shows the peak corresponding to lattice planes $(400)$ together with calculated peak positions for theoretical bulk lattice parameters of $\gamma-\mathrm{Fe}_{2} \mathrm{O}_{3}$ and $\mathrm{Fe}_{3} \mathrm{O}_{4}$. Peaks are indexed with the cubic spinel structure. ing according to $B_{h k l}={\sqrt{B_{\text {obs }}^{2}-B_{\text {standard }}^{2}}}^{66}$ with the NIST standard 660a $\mathrm{LaB}_{6}$.

The FWHM for the peaks (220), (311) and (511) are approximately doubled compared to the ones obtained from (222), (440) and (400) (Table 2). A similar result was reported by Wetterskog et al. ${ }^{27}$ based on peak width analysis of 220, 440 and 400 peaks in iron oxide nanoparticles. This indicates that the structurally coherent domain size is reduced in the direction perpendicular to the (220), (311) and (511) lattice planes. The orientation of the planes corresponding to the (220) peak is the same as that of the shifting plane associated with the APB. The lattice planes (311) and (511) are not completely parallel to this translation, however they are only slightly tilted with respect to the (110) plane. The (440) peak is not influenced by this antiphase boundary lattice shift, in spite of being parallel to the shifting plane, because the corresponding plane distance is smaller than the region of the unit cell affected by the translation. This can be illustrated by Fourier transforming a micrograph of a nanoparticle and masking the respective Bragg peaks before performing the inverse Fourier transform as shown in Fig. 3(c) and (d). The effect of an APB is only visible in the image with the (220) peak masked. The approximate doubling of the FWHM for planes parallel to the APB leads to an approximate halving of the coherent crystallite, which is to be expected considering that the observed XRD pattern represents an average over many particles and particle orientations. If APBs develop with different distances to the particle surface, the average distance will be approximately half the particle diameter. The fact that we clearly observe such doubling of the respective FWHM indicates that a large portion of particles contains APBs.

As previously mentioned the exchange bias effect visible in inset in Fig. 2(b) could also be attributed to a ferro(ferri)magnetic/antiferromagnetic interface, e.g. the presence of wüstite $(\mathrm{FeO})$ in maghemite/magnetite $\left(\gamma-\mathrm{Fe}_{2} \mathrm{O}_{3} / \mathrm{Fe}_{3} \mathrm{O}_{4}\right)$ particles. ${ }^{55,56}$ The Néel temperature of wüstite (FeO) is $198 \mathrm{~K}$ (ref. 67) and is thus obscured by the superparamagnetic transition. For magnetite $\left(\mathrm{Fe}_{3} \mathrm{O}_{4}\right)$ a shoulder or a kink in the ZFC curve of a $M(T)$ measurement would be expected near the Verwey transition temperature $T_{\mathrm{V}}=120 \mathrm{~K} .{ }^{68}$ However, in the experimental ZFC curve no feature around the Verwey temperature was observed. According to Goya et al. ${ }^{69}$ such transition can be suppressed for sufficiently small particles (diameter $<50 \mathrm{~nm}$ ). An exact

Table 2 Peak width analysis of the peaks shown in Fig. 4

\begin{tabular}{lllr}
\hline$h k l$ & $Q\left(\AA^{-1}\right)$ & FWHM $\left(^{\circ}\right)$ & $D_{h k l}(\AA)$ \\
\hline 220 & $2.126(1)$ & $0.33(1)$ & $71(1)$ \\
311 & $2.487(1)$ & $0.30(1)$ & $78(1)$ \\
222 & $2.598(1)$ & $0.15(1)$ & $152(1)$ \\
400 & $3.007(1)$ & $0.16(1)$ & $149(1)$ \\
422 & $3.683(1)$ & $0.37(1)$ & $63(1)$ \\
511 & $3.907(1)$ & $0.34(1)$ & $69(1)$ \\
440 & $4.253(1)$ & $0.18(1)$ & $135(1)$ \\
$K=0.94, \lambda=0.4329 \AA$. & &
\end{tabular}


knowledge about the particle composition is of great importance for a proper assessment of the origin of the exchange bias and can be obtained from the analysis of the pair distribution function, derived from the X-ray total scattering spectrum. It has to be taken into account that three superstructure peaks appear at $1.51,1.68$ and $1.85 \AA^{-1}$ (marked with asterisks in Fig. 4), which are related to a vacancy ordering mainly on the octahedral sites and a resulting reduction of the symmetry from a cubic to a tetragonal structure. ${ }^{70-72}$

The PDF represents a probability of atoms to be separated by the distance $r$ (Fig. 5) and provides insight into the particle composition and the local crystal structure. It is obtained by a Fourier transformation of the total scattering structure function, $S(Q)$, which is the background corrected and normalized synchrotron powder scattering data $I(Q) \cdot{ }^{73}$ For the refinement a crystal structure model for maghemite with the space group $P 4_{3} 2_{1} 2$ with a tetragonal/pseudocubic unit cell as proposed in the literature ${ }^{72,74,75}$ was used as a starting point. As stated above, this choice is supported by the presence of the superstructure peaks in the diffraction pattern (Fig. 4). A thorough discussion of this space group choice is presented in the ESI (ESI Fig. S1, S2 and Table S1 $\dagger$ ). Refinement parameters are the lattice constants $a=b$ and $c$, the atomic positions, a scale factor, a factor accounting for temperature effects affecting the low $r$ region $(\delta)$ and site occupancy factors (SOFs) of the iron
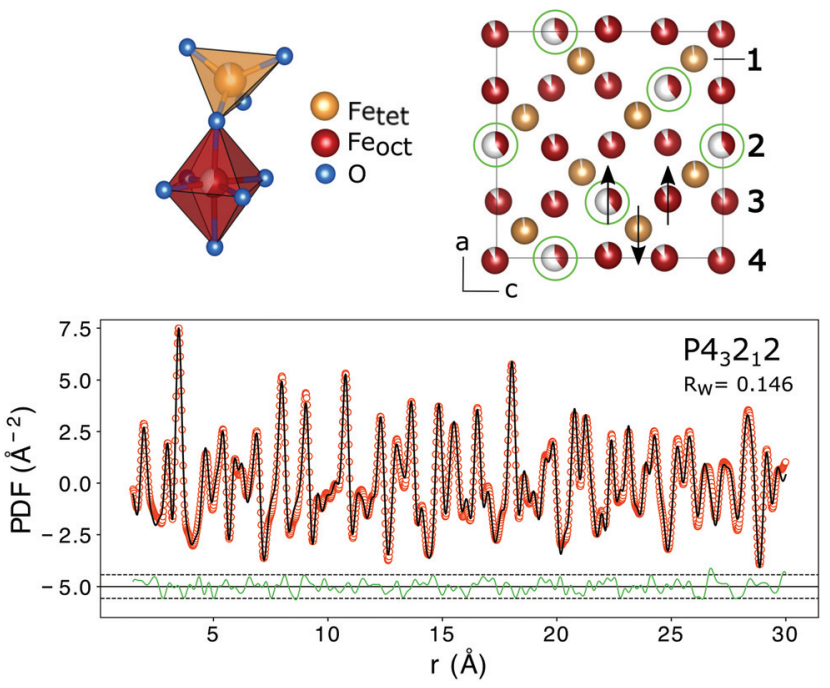

Fig. 5 The top left shows the structural units with the iron atoms either octahedrally or tetrahedrally coordinated. Vacancy ordering on the iron positions is indicated with green circles in the top right figure. The numbers correspond to the nomenclature of the iron positions used in Table 3. The arrows show the atomic spin vectors expected for the bulk material leading to a ferrimagnetic structure. In the lower figure the PDF fit of interatomic distances $r$ from 1.5 to $16 \AA$ using a tetragonal/pseudocubic crystal structure with space group symmetry $P 4_{3} 2_{1} 2$ is shown. Interatomic distances smaller than the first coordination sphere were not included in the fit. The dashed lines represent 2 standard deviations of the difference curve. The structural parameters obtained from the fit are listed in Table 3.
Table 3 Results of the PDF analysis presented in Fig. 5. Isotropic displacement parameters $\left(U_{\text {iso }}\right)$ for the oxygen atoms were constrained. The position designation is according to the crystal structure drawn in Fig. 5. Particle size corresponds to the effective diameter of the particles, which is reduced compared to the value from SAXS due to the above mentioned selective peak broadening that enters the PDF through the Fourier transform of the total X-ray scattering pattern

\begin{tabular}{llllll}
\hline Position & $x / a$ & $y / b$ & $z / c$ & $U_{\text {iso }}$ & SOF \\
\hline Fe1(tet.) & $0.7469(5)$ & $0.9985(5)$ & $0.1201(5)$ & $0.0068(5)$ & $0.98(2)$ \\
Fe2(oct.) & $0.3672(5)$ & $0.6328(5)$ & 0.75 & $0.0073(5)$ & $0.39(2)$ \\
Fe3(oct.) & $0.1205(5)$ & $0.8795(5)$ & 0.25 & $0.0069(5)$ & $0.93(2)$ \\
Fe4(oct.) & $0.3685(5)$ & $0.8683(5)$ & $0.9866(5)$ & $0.0067(5)$ & $0.92(2)$ \\
O1 & $0.1414(5)$ & $0.3833(5)$ & $0.5077(5)$ & $0.0119(5)$ & 1 \\
O2 & $0.3794(5)$ & $0.1314(5)$ & $0.0013(5)$ & $0.0119(5)$ & 1 \\
O3 & $0.1299(5)$ & $0.8676(5)$ & $0.0232(5)$ & $0.0119(5)$ & 1 \\
O4 & $0.3749(5)$ & $0.6293(5)$ & $0.9933(5)$ & $0.0119(5)$ & 1
\end{tabular}

$a=b=8.358(2) \AA, c=8.358(2) \AA . \quad \delta=1.66(1) \AA^{-1}$, particle size $=106(5)$ $\AA, Q_{\max }=18 \AA^{-1}, r_{\text {poly }}=1.3 \AA, R_{\mathrm{w}}=0.146$.

positions. The fit results are shown in Table 3. The X-ray scattering spectrum clearly shows a mixed phase composition of the nanoparticles (inset in Fig. 4). The dominant phase is maghemite $\left(\gamma-\mathrm{Fe}_{2} \mathrm{O}_{3}\right)$ with a smaller contribution of magnetite $\left(\mathrm{Fe}_{3} \mathrm{O}_{4}\right)$. Wüstite $(\mathrm{FeO})$ should produce a strong peak at around $Q=2.91 \AA^{-1}$, which is not present in the observed pattern. The lattice parameters obtained from the PDF analysis can be used to quantify this phase composition. The average lattice parameter of $8.358 \AA$ corresponds to roughly $18(10) \% \mathrm{Fe}_{3} \mathrm{O}_{4}$. The error of this estimate is quite large due to large deviations of the bulk $\gamma-\mathrm{Fe}_{2} \mathrm{O}_{3}$ lattice parameters in the literature. ${ }^{76}$ We like to note that in order to exclude a possible influence of the instrument calibration on these measured values bulk reference samples of $\mathrm{Fe}_{3} \mathrm{O}_{4}$ and $\mathrm{FeO}$ and a nanopowder with a mix of $\mathrm{Fe}_{3} \mathrm{O}_{4}$ and $\gamma-\mathrm{Fe}_{2} \mathrm{O}_{3}$ (50 to $100 \mathrm{~nm}$ in size) have been measured. An independent estimation of the particle phase composition can be obtained using Mössbauer spectroscopy (Fig. 6). The Mössbauer spectrum consists of three sextet subspectra, assigned to B-site $\mathrm{Fe}^{3+}$ (dark red), A-site $\mathrm{Fe}^{3+}$ (red) and B-site $\mathrm{Fe}^{2+}$ (orange). Due to the limited thermal energy at $4.3 \mathrm{~K}$, no signs of beginning superparamagnetic relaxation are visible, wherefore the subspectra could be reproduced via symmetric sextets. Due to the lower magnetic moment of $\mathrm{Fe}^{2+}$ as compared to $\mathrm{Fe}^{3+}$, the $\mathrm{Fe}^{2+}$ subspectrum exhibits a smaller sextet splitting, as well as a higher isomer shift due to different electronic charge densities close to the iron nucleus. ${ }^{77,78}$ To obtain higher precision in the estimation of the $\mathrm{Fe}^{2+}$ fraction, hyperfine parameters of the three sextet components were compared to those of larger particles obtained from the identical synthesis route, exhibiting higher contents of $\mathrm{Fe}_{3} \mathrm{O}_{4} . \mathrm{Fe}^{2+}$ is only present in the $\mathrm{Fe}_{3} \mathrm{O}_{4}$ structure and can therefore be used to estimate the phase composition, yielding a $\mathrm{Fe}_{3} \mathrm{O}_{4}$ contribution of roughly $15 \%$ with the rest being $\gamma-\mathrm{Fe}_{2} \mathrm{O}_{3}$, in agreement to lattice constants of the particles discussed above. A FeO (wüstite) contribution is not apparent in the spectrum, thus also ruling out $\mathrm{FeO}$ as the cause for the 


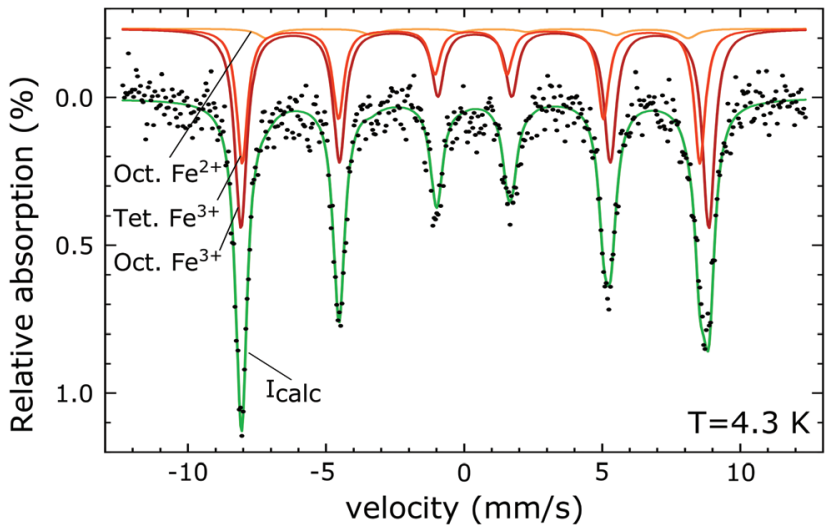

Fig. 6 Mössbauer spectrum recorded at $4.3 \mathrm{~K}$. The fit curve (green) is obtained by a superposition of three magnetic sextets, corresponding to $\mathrm{Fe}^{3+}$ on octahedral and tetrahedral positions and $\mathrm{Fe}^{2+}$ on octahedral sites.

visible exchange bias in Fig. 2(b) and further supporting the presence of APBs as stated earlier.

In addition to the particle composition, the PDF allows also the investigation of the local crystal structure as presented in the following. The refined crystal lattice features slightly distorted coordination polyhedra and the refinement of SOFs reveals partial vacancy ordering with the majority of vacancies on octahedral Fe2 lattice sites (Fig. 5). The preference of vacancies for octahedral lattice sites could be a result of the oxidation of $\mathrm{Fe}_{3} \mathrm{O}_{4}$ to $\gamma-\mathrm{Fe}_{2} \mathrm{O}_{3}$. Assuming an in situ oxidation of $\mathrm{Fe}^{2+}$-ions to $\mathrm{Fe}^{3+}$ through the migration of electrons a charge imbalance will form locally which drives the $\mathrm{Fe}_{3}{ }^{+}$-ion outwards, leaving a vacancy behind. Since $\mathrm{Fe}^{2+}$ is situated only on octahedral lattice sites in the $\mathrm{Fe}_{3} \mathrm{O}_{4}$ structure due to its larger ionic radius, the vacancies will be mainly formed on octahedral sites. ${ }^{79}$ The vacancy ordering is not complete, as a reduced occupancy is also found on the tetrahedral iron position Fe1 and on the other octahedral iron sites (Table 3). In total, the amount of iron atoms in the unit cell corresponds to an occupation of $85(2) \%$, which is slightly lower than the theoretical value of $88.9 \%$ for maghemite $\left(\gamma-\mathrm{Fe}_{2} \mathrm{O}_{3}\right) .{ }^{71}$ This leads to a theoretic reduction of the saturation magnetization by $9 \%$, assuming perfect parallel and antiparallel alignment of atomic moments with an applied field on octahedral and tetrahedral positions and magnetic moments of 4.19 and $4.03 \mu_{\mathrm{B}}$, respectively. ${ }^{71}$ This is, however, neglecting the influence of vacancies on the local environment and thus the exchange interactions. The average iron-oxygen distances for the vacancy rich Fe2 position $(2.119 \AA)$ are larger than for the other two octahedral positions (1.981 $\AA$ for Fe3 and $2.064 \AA$ for Fe4), which shows the influence of the vacancies on the local environment. The super-exchange interactions present between the iron atoms are highly dependent on the bond lengths and angles. ${ }^{58}$ In the refined crystal structure the $\mathrm{Fe}-\mathrm{O}-\mathrm{Fe}$ bond-angles between tetrahedral and octahedral iron are in the range of 114 to $130^{\circ}$ giving rise to the antiferromagnetic coupling of both sublat- tices. The moments of octahedrally coordinated iron atoms couple ferromagnetically with an average $\mathrm{Fe}-\mathrm{O}-\mathrm{Fe}$ bond angle of $90^{\circ}$. However in the vicinity of a vacancy position (Fe2) some bond angles between adjacent iron atoms are altered to $102^{\circ}$. This would probably weaken the ferromagnetic alignment and lead to a reduction of the macroscopic magnetization. On the other hand it was previously shown that vacancy ordering, as observed in the samples of this study, increases the magnetization of iron oxide nanoparticles compared to particles with disordered vacancies, ${ }^{80}$ even up to bulk values for particles with a high degree of vacancy ordering and otherwise defect free crystal structures. ${ }^{81}$ Additionally, with the small-box approach used in PDFGUI, i.e. the assumption of periodic boundary conditions, it is not possible to model large range variations in the particle composition or vacancy concentrations. It might be that the increased number of vacancies is linked to structural disorder at the particle surface. Atomistic simulations that might determine the influence of vacancies on the saturation magnetization more precisely are very complex and beyond the scope of this work. However, we can still conclude that the partial vacancy disorder contributes to the observed reduction in magnetization, but alone is not sufficient to explain it in full.

The $M(H)$ curves in Fig. 2(b) were fitted with the Langevin function under consideration of the particle size distribution $^{50,82}$ as determined from SAXS

$$
M(B, T)=\int_{0}^{\infty} M_{\mathrm{sat}} L\left(\frac{\mu_{\mathrm{p}} B}{k_{\mathrm{B}} T}\right) P(v) \mathrm{d} v .
$$

Here, $\mu_{\mathrm{p}}$ is the particle magnetic moment, which is related to the saturation magnetization via $M_{\mathrm{sat}}=\mu_{\mathrm{p}} / V_{\mathrm{t}} \rho$, where $V_{\mathrm{t}}$ is the total particle volume and $\rho$ the particle density. $P(v)$ is the particle size distribution, as given in eqn (1). $B$ is the magnetic induction, $k_{\mathrm{B}}$ and $T$ are the Boltzmann constant and the temperature, respectively. $L(x)$ is the Langevin function according to

$$
L(x)=\operatorname{coth}(x)-\frac{1}{x}
$$

At $300 \mathrm{~K}$ the saturation magnetization constituted 83(1) A $\mathrm{m}^{2} \mathrm{~kg}_{\mathrm{Fe}}^{-1}$ (using the amount of iron of 1.363(17) $\mathrm{g}$ as obtained by ICP-OES (ESI Table S1†)). Assuming either pure $\mathrm{Fe}_{3} \mathrm{O}_{4}$ or $\gamma-\mathrm{Fe}_{2} \mathrm{O}_{3}$ this yields 61(1) $\mathrm{A} \mathrm{m}^{2} \mathrm{~kg}_{\mathrm{Fe}_{3} \mathrm{O}_{4}}{ }^{-1}$ and 59(1) $\mathrm{A} \mathrm{m}^{2}$ $\mathrm{kg}_{\gamma-\mathrm{Fe}_{2} \mathrm{O}_{3}}{ }^{-1}$, respectively. Considering the particle composition a value of 60 (1) $\mathrm{A} \mathrm{m}^{2} \mathrm{~kg}_{\mathrm{Ferrite}}{ }^{-1}$ is obtained. The values for the saturation magnetization are significantly lower than the room temperature bulk values for $\mathrm{Fe}_{3} \mathrm{O}_{4}\left(86 \mathrm{~A} \mathrm{~m}^{2} \mathrm{~kg}^{-1}\right.$ (ref. 83)) and $\gamma-\mathrm{Fe}_{2} \mathrm{O}_{3}\left(75 \mathrm{~A} \mathrm{~m}^{2} \mathrm{~kg}^{-1}\right.$ (ref. 84)) suggesting a decrease in saturation magnetization of about $23 \%$. By referring to room temperature data the temperature dependence of the saturation magnetization is implicitly taken into account. Comparison to bulk values is justified through the observation of nearly bulk saturation magnetization values in iron oxide nanoparticles in previous studies. ${ }^{29,83,85}$

In order to study the magnetic structure of the nanoparticles a series of SANS experiments with polarized neutrons 
was performed where the scattering intensities for the two neutron spin states are given by

$$
\begin{gathered}
I^{-}(Q, \alpha)={F_{\mathrm{N}}}^{2}+\left({F_{\mathrm{M}}}^{2}-2 P F_{\mathrm{N}} F_{\mathrm{M}}\right) \sin ^{2} \alpha, \\
I^{+}(Q, \alpha)={F_{\mathrm{N}}}^{2}+\left(F_{\mathrm{M}}^{2}+2 P \varepsilon F_{\mathrm{N}} F_{\mathrm{M}}\right) \sin ^{2} \alpha,
\end{gathered}
$$

where $P$ is the incident neutron polarization, $\varepsilon$ is the flipper efficiency and $\alpha$ is the angle in detector plane between the $Q$ vector and the direction of the applied magnetic field. ${ }^{86} F_{\mathrm{N}}$ and $F_{\mathrm{M}}$ are the nuclear and magnetic form factors, respectively. Superscripts - and + correspond to neutron spin state antiparallel to the magnetic field (spin down) and parallel to it (spin up). For $Q \| H$, i.e. when $\alpha=0^{\circ}$ or $\alpha=180^{\circ}$, the term in parentheses in eqn (6) and (7) does not contribute and the scattering intensity results purely from to the nuclear form factor. ${ }^{86-88}$ To improve statistics a sector of $10^{\circ}$ width was integrated around $0^{\circ}$ and $180^{\circ}$. The data in sectors obtained in such a way were averaged between up and down channels for each contrast. A simultaneous fit of the five obtained scattering curves to a model of a core-shell sphere was performed in a combination with a sticky hard sphere structure factor that accounts for the weak interaction between the nanoparticles detected along the direction of the applied magnetic field (Fig. 7(a)). Using parameters of the lognormal size distribution from SAXS the thickness of the oleic acid shell was found to be 1.4(1) $\mathrm{nm}$, which is in agreement with previously reported values. $^{26,89,90}$ Extrapolated SANSPOL intensities at $Q=0$ plotted against the relative deuteration reveal the match point, i.e. the point where the scattering intensity takes its minimum due to the proximity of the solvent SLD to the average SLD of the nanoparticles (Fig. 7(b)). Following Avdeev $^{90}$ with the knowledge of the lognormal size distribution the total $\left(V_{\mathrm{t}}\right)$, core $\left(V_{\mathrm{c}}\right)$ and shell $\left(V_{\mathrm{s}}\right)$ volume, as well as the scattering length density of the shell $\left(\mathrm{SLD}_{\mathrm{S}}\right)$ it is possible to calculate the core $\operatorname{SLD}\left(\mathrm{SLD}_{\mathrm{c}}\right)$ according to

$$
\operatorname{SLD}_{\mathrm{c}}=\frac{\operatorname{SLD}_{\mathrm{mp}}\left\langle V_{\mathrm{t}}^{2}\right\rangle-\operatorname{SLD}_{\mathrm{s}}\left\langle V_{\mathrm{s}} V_{\mathrm{t}}\right\rangle}{\left\langle V_{\mathrm{c}} V_{\mathrm{t}}\right\rangle},
$$

where angle brackets symbolize averaging over the particle size distribution (eqn (1)). With $\mathrm{SLD}_{\mathrm{s}}=0.08 \times 10^{-6} \AA^{-2}$ and the match point at $69(2) \%$ d-toluene this results in a nuclear core SLD of $6.8(2) \times 10^{-6} \AA^{-2}$, which is in excellent agreement with the theoretical value of $6.7 \times 10^{-6} \AA^{-2}$ assuming the composition as determined by PDF analysis and Mössbauer spectroscopy. The scattering intensity of the SANSPOL experiment in the sector perpendicular to the magnetic field direction was used to get information on the magnetization distribution within the nanoparticle. We observed no signs of interparticle interaction in the direction perpendicular to magnetic field, i.e. no structure factor contribution to the scattered intensity. Subtraction of $I$ from $I^{+}$given in eqn (6) and (7) results in the magnetic-nuclear interference term $B_{\text {cross }}$ (Fig. 7c),

$$
\begin{aligned}
I^{+}(Q, \alpha)-I^{-}(Q, \alpha)= & 2 P(1+\varepsilon) F_{\mathrm{N}} F_{\mathrm{M}} \sin ^{2} \alpha \\
& =B_{\text {cross }}(Q) \sin ^{2} \alpha .
\end{aligned}
$$

A simultaneous fit of all five interference terms was performed. For $F_{\mathrm{N}}$ the core-shell model was used with all parameters fixed from the analysis of scattering intensity in sectors parallel to the applied magnetic field. In case of $F_{\mathrm{M}}$ a coreshell model with magnetic SLDs was used. The only fit parameters were a non-magnetic shell thickness, $t_{\text {mag, }}$ and the magnetic scattering length density, $\mathrm{SLD}_{\mathrm{m}}$. The shell thickness evaluated to $0.3(1) \mathrm{nm}$ giving a $\mathrm{SLD}_{\mathrm{m}}$ for the magnetic core of $9.4(2) \times 10^{-7} \AA^{-2}$ (a detailed description of the fitting procedure is presented in the ESI $\dagger$ ). The magnetic scattering length density $\mathrm{SLD}_{\mathrm{m}}$ is related to the magnetic particle moment
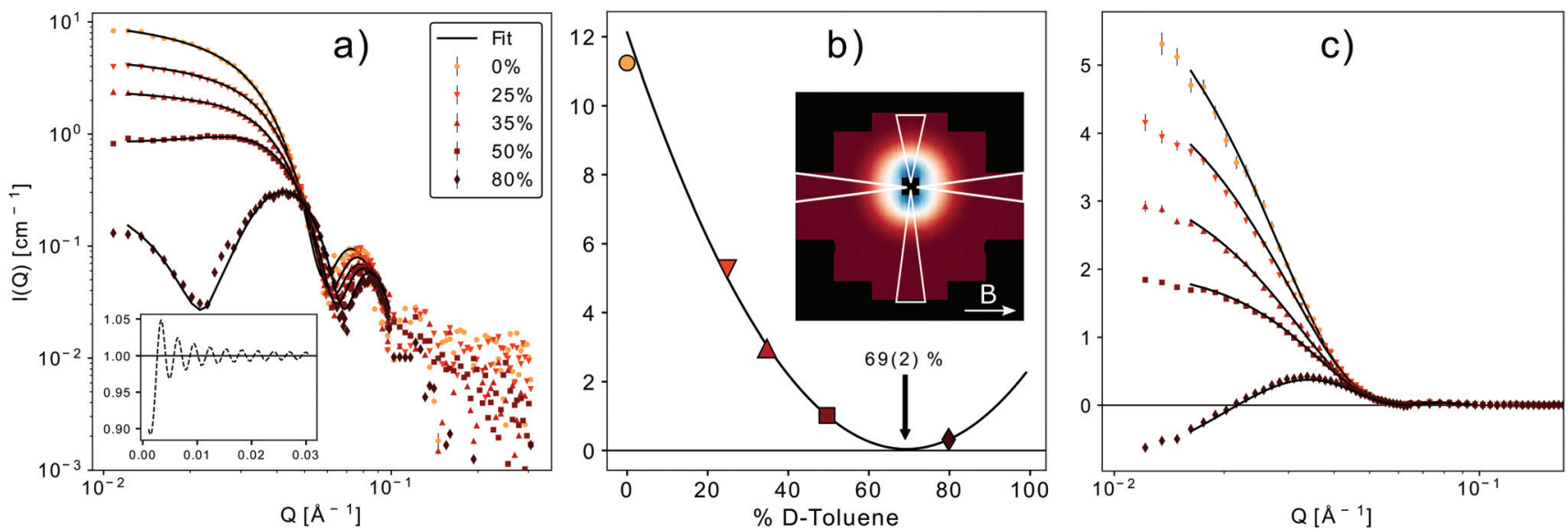

Fig. 7 (a) SANSPOL with 5 different contrasts. The black line represents the best fit of a core-shell model with a sticky hard sphere structure factor (inset on the lower left). (b) Contrast matching. The minimum of the parabolic fit corresponds to the match point resulting in a core SLD of $6.8(2) \times$ $10^{-6} \AA^{-2}$. The inset shows the $2 \mathrm{D}$-detector image for $50 \% \mathrm{~d}$-toluene in the up-channel. The white triangles mark the integration sectors. (c) Fit of the magnetic-nuclear interference terms for the 5 contrasts, according to eqn (9). The only fit parameters are the magnetic scattering length density $\left(S L D_{m}\right)$ and thickness of a magnetically dead shell. 
according to $\mu_{\mathrm{p}}=\mathrm{SLD}_{\mathrm{m}} V_{\text {eff }} \mu_{\mathrm{B}} / C$, where $\mu_{\mathrm{B}}$ is the Bohr magneton and $C$ is a constant given by $C=1 / 2 g_{\mathrm{n}} r_{0}=2.7 \times 10^{-5} \AA$, with $g_{\mathrm{n}}$ the neutron gyromagnetic factor, $r_{0}$ the classical electron radius. $V_{\text {eff }}$ is the effective magnetic particle volume. ${ }^{26}$ Together with $M_{\text {sat }}=\mu_{\mathrm{p}} / V_{\mathrm{t}} \rho$ the saturation magnetization $M_{\mathrm{sat}}$ in $\mathrm{A} \mathrm{m}^{2} \mathrm{~kg}^{-1}$ is related to $\mathrm{SLD}_{\mathrm{m}}$ in $\AA^{-2}$ via

$$
M_{\mathrm{sat}}=\frac{\mathrm{SLD}_{\mathrm{m}} \mu_{\mathrm{B}}}{C \rho} \frac{V_{\mathrm{eff}}}{V_{\mathrm{t}}},
$$

where $\rho$ is the mass density and $V_{\mathrm{t}}$ is the total particle volume.

From $\mathrm{SLD}_{\mathrm{m}}$ a saturation magnetization of $58(4) \mathrm{A} \mathrm{m}^{2}$ $\mathrm{kg}_{\text {Ferrite }}{ }^{-1}$ is calculated, which is in very good agreement to $M_{\text {sat }}$ at $300 \mathrm{~K}$ from the SQUID-magnetometry measurements that constituted 59(1) $\mathrm{A} \mathrm{m}^{2} \mathrm{~kg}_{\text {Ferrite }}{ }^{-1}$ (Fig. 2b).

In the work of Disch et l. $^{26}$ spherical particles with diameter of $9.94 \mathrm{~nm}$ and FWHM of a lognormal size distribution of 0.055 were used. The authors concluded based on SANSPOL, VSM, SAXS and TEM data that a small magnetically depleted surface layer of $0.3(1) \mathrm{nm}$ is present, but the main factor leading to the observed reduction of the magnetization compared to bulk values must be effects in the nanoparticle core in line with our findings. In the work of Herlitschke et $a .^{28}$ no magnetic core-shell structure was reported for spherical particles with diameter of $7.4 \mathrm{~nm}$ and $\sigma$ of a lognormal size distribution of 0.057 and the reduction of the magnetization was entirely ascribed to random orientations of atomic moments homogeneously distributed in the particle. A recent study by Zákutná et al. ${ }^{91}$ on cobalt ferrite nanoparticles with mean diameter of $14.1 \mathrm{~nm}$ and lognormal size distribution of $\sigma=0.031$ revealed a magnetically disordered surface region of $0.7 \mathrm{~nm}$ thickness. Since the particles used there were composed of cobalt ferrite the results are only partially comparable to pure ferrite nanoparticles. In the work of Krycka et al. ${ }^{24}$ nanoparticles with diameter of $9.0 \mathrm{~nm}$ were investigated by SANS with polarization analysis. Here a magnetic surface layer of 1.0(2) nm with magnetization direction orthogonal to the core was proposed. Measurements were done on self assembled particles with considerable interparticle interactions that might influence this result and make comparisons to our samples difficult. Sharifi Dehsari et al. ${ }^{30}$ investigated particles ranging in diameter from 6.3 to $16.2 \mathrm{~nm}$ and found that particles consist of both magnetite and maghemite phases with possibly magnetic domains for particles larger than $14 \mathrm{~nm}$ and concluded from fittings to $M(H)$ curves that no magnetic dead layer is present in all particle sizes. These inconsistent results with regards to a non-magnetic surface layer reflect the difficulties in assessing the particle surface with experimental methods and the data analysis. However, most authors agree that intraparticle effects play a major role in the reduction of saturation magnetization regardless of the presence of a surface layer.

A non-magnetic surface layer of $0.3 \mathrm{~nm}$ thickness could account for a magnetization reduction of $\max .10 \%$. Considering that the magnetization is $23 \%$ lower in the particles of this study compared to bulk reference values a surface layer alone is not sufficient to explain the reduction. The observed increased amount of vacancies in the crystal structure might also reduce the magnetization, however, as mentioned above, the observed ordering of the vacancies counterbalances this effect. Additionally, vacancies might preferably form on the particle surface and lead to the small non-magnetic surface layer that was observed with neutron scattering. The obtained data point to the fact that the observed magnetization reduction stems mainly from the core of the nanoparticles, i.e. due to the presence of antiphase boundaries.

Finally, the Langevin fit to the $300 \mathrm{~K} M(H)$ data yields a magnetic particle moment of $\mu_{\mathrm{p}}=63303 \mu_{\mathrm{B}}$, consistent with the $\mu_{\mathrm{p}}$ obtained from SANS $\left(\mu_{\mathrm{p}}=63102 \mu_{\mathrm{B}}\right)$. However, a closer examination of the fit curve shows a deviation from the data for field values between -0.2 and $0.2 \mathrm{~T}$ (Fig. 8), which would be better described by a smaller particle moment. In order to achieve the same saturation magnetization such a small particle moment would require a reduced effective magnetic particle radius of $6.8 \mathrm{~nm}$, much smaller than the magnetic radius of $7.5 \mathrm{~nm}$ we observed in SANS. More likely are either a distribution of magnetic moments ${ }^{30}$ or a field dependence of the particle magnetic moment, originating from a field dependence of the magnetic particle volume. The magnetically depleted surface layer could increase for decreasing fields, as was observed for cobalt ferrite nanoparticles. ${ }^{91}$ In addition the region of canted spins in the vicinity of the APB also increases for decreasing fields, as observed in iron oxide thin films. ${ }^{92}$ Both effects will reduce the magnetic volume of the particle, and lead to a smaller magnetic moment for smaller fields.

In summary we were able to assess the structural, chemical and magnetic properties of the iron oxide nanoparticles used in this study. Our findings are compiled in the following:

- Reduced room-temperature saturation magnetization of 60(1) $\mathrm{A} \mathrm{m}^{2} \mathrm{~kg}_{\text {Ferrite }}{ }^{-1}$ compared to the bulk materials

- Core size of 7.8(1) $\mathrm{nm}$ and $\sigma=0.095(1)$ of a lognormal size distribution and organic ligand shell thickness of $1.4(1) \mathrm{nm}$

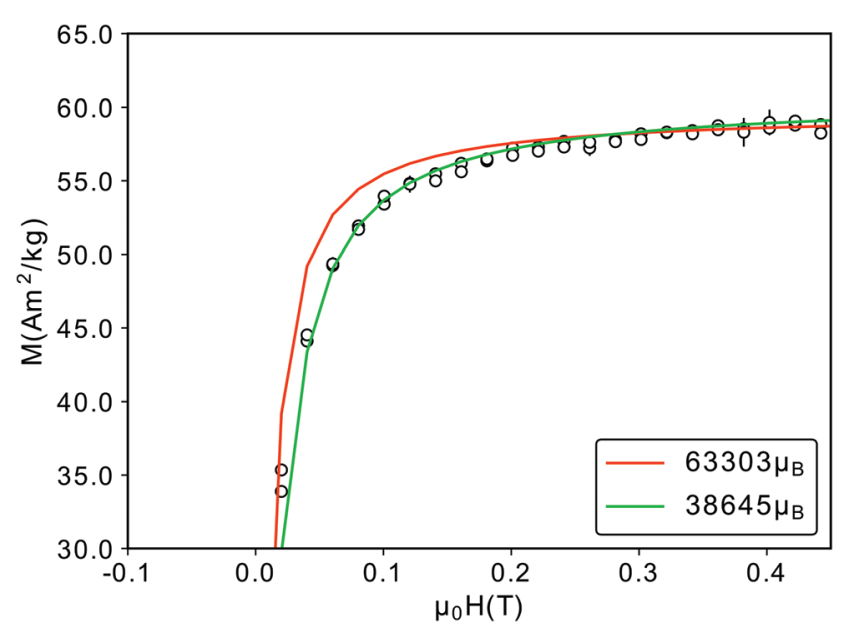

Fig. 8 Close-up of the $M(H)$-RT data depicted in Fig. 2(b). The red curve corresponds to a magnetic particle moment of $63303 \mu_{\mathrm{B}}$, a better fit is achieved with a smaller particle moment (green). 
- Particles are composed of ca. $15 \% \quad \mathrm{Fe}_{3} \mathrm{O}_{4}$ and $85 \%$ $\gamma-\mathrm{Fe}_{2} \mathrm{O}_{3}$, with no considerable contribution of $\mathrm{FeO}$ or other phases

- A magnetically dead surface layer of $0.3(1) \mathrm{nm}$ was found

- Slightly increased number of vacancies, with partial order, thereby reducing the crystal symmetry to a tetragonal space group

- Antiphase boundaries are found directly and indirectly by HR-TEM and X-ray total scattering.

\section{Conclusions}

In conclusion, to resolve the open question about the underlying mechanism leading to the reduction of magnetization in iron oxide nanoparticles we addressed aspects such as particle size, size distribution, composition, structural and magnetic properties. Our results suggest that the observed reduced magnetization is a consequence of primarily the presence of antiphase boundaries in the nanoparticle core. We presented a consistent and precise analysis of the particle surface and the internal structural defects. For potential improvements of the particle performance in magnetic applications we propose to focus on the elimination of intraparticle effects.

\section{Conflicts of interest}

There are no conflicts to declare.

\section{Acknowledgements}

We acknowledge the Paul Scherrer Institut, Villigen, Switzerland for provision of synchrotron radiation beamtime at beamline MS-X04SA of the SLS. AK and RDB acknowledge the financial support to European Union's Horizon 2020 research and innovation programme (Grant No. 856538) and to the Deutsche Forschungsgemeinschaft (Pr. 405553726 - TRR 270). This work is based upon experiments performed at the KWS-1 instrument operated by JCNS at the Heinz MaierLeibnitz Zentrum (MLZ), Garching, Germany.

\section{References}

1 R. Bailey, J. Magn. Magn. Mater., 1983, 39, 178-182.

2 K. Raj, B. Moskowitz and R. Casciari, J. Magn. Magn. Mater., 1995, 149, 174-180.

3 Y.-P. Sun, Supercritical Fluid Technology in Materials Science and Engineering: Syntheses: Properties, and Applications, Crc Press, 2002.

4 L. Josephson, C.-H. Tung, A. Moore and R. Weissleder, Bioconjugate Chem., 1999, 10, 186-191.

5 R. C. Semelka and T. K. Helmberger, Radiology, 2001, 218, $27-38$.
6 C. Corot, P. Robert, J.-M. Idée and M. Port, Adv. Drug Delivery Rev., 2006, 58, 1471-1504.

7 Y.-W. Jun, J.-H. Lee and J. Cheon, Angew. Chem., Int. Ed., 2008, 47, 5122-5135.

8 S. Laurent, D. Forge, M. Port, A. Roch, C. Robic, L. Vander Elst and R. N. Muller, Chem. Rev., 2008, 108, 2064-2110.

9 C. Sun, J. S. Lee and M. Zhang, Adv. Drug Delivery Rev., 2008, 60, 1252-1265.

10 X. Ma, A. Gong, B. Chen, J. Zheng, T. Chen, Z. Shen and A. Wu, Colloids Surf., B, 2015, 126, 44-49.

11 R. Hergt, S. Dutz, R. Müller and M. Zeisberger, J. Phys.: Condens. Matter, 2006, 18, S2919.

12 M. Gonzales-Weimuller, M. Zeisberger and K. M. Krishnan, J. Magn. Magn. Mater., 2009, 321, 1947-1950.

13 K. M. Krishnan, IEEE Trans. Magn., 2010, 46, 2523-2558.

14 C. S. Kumar and F. Mohammad, Adv. Drug Delivery Rev., 2011, 63, 789-808.

15 S. Laurent, S. Dutz, U. O. Häfeli and M. Mahmoudi, Adv. Colloid Interface Sci., 2011, 166, 8-23.

16 A. E. Deatsch and B. A. Evans, J. Magn. Magn. Mater., 2014, 354, 163-172.

17 R. L. White, R. Newt and R. F. W. Pease, IEEE Trans. Magn., 1997, 33, 990-995.

18 C. Jiang, S. M. Ng, C. W. Leung and P. W. T. Pong, J. Mater. Chem. C, 2017, 5, 252-263.

19 E. Tronc, Adv. Chem. Phys., 1997, 283, 283-449.

20 S. Majetich and Y. Jin, Science, 1999, 284, 470-473.

21 A. Roca, D. Niznansky, J. Poltierova-Vejpravova, B. Bittova, M. Gonzalez-Fernandez, C. Serna and M. Morales, J. Appl. Phys., 2009, 105, 114309.

22 T. J. Daou, J.-M. Greneche, S.-J. Lee, S. Lee, C. Lefevre, S. Bégin-Colin and G. Pourroy, J. Phys. Chem. C, 2010, 114, 8794-8799.

23 O. Petracic, Superlattices Microstruct., 2010, 47, 569-578.

24 K. L. Krycka, R. A. Booth, C. R. Hogg, Y. Ijiri, J. A. Borchers, W. Chen, S. Watson, M. Laver, T. R. Gentile, L. R. Dedon, S. Harris, J. J. Rhyne and S. A. Majetich, Phys. Rev. Lett., 2010, 104, 207203.

25 F. Kronast, N. Friedenberger, K. Ollefs, S. Gliga, L. TatiBismaths, R. Thies, A. Ney, R. Weber, C. Hassel, F. M. Römer, A. V. Trunova, C. Wirtz, R. Hertel, H. A. Dürr and M. Farle, Nano Lett., 2011, 11, 1710-1715.

26 S. Disch, E. Wetterskog, R. P. Hermann, A. Wiedenmann, U. Vainio, G. Salazar-Alvarez, L. Bergström and T. Brückel, New J. Phys., 2012, 14, 013025.

27 E. Wetterskog, C.-W. Tai, J. Grins, L. Bergström and G. Salazar-Alvarez, ACS Nano, 2013, 7, 7132-7144.

28 M. Herlitschke, S. Disch, I. Sergueev, K. Schlage, E. Wetterskog, L. Bergström and R. P. Hermann, J. Phys.: Conf. Ser., 2016, 711, 012002.

29 Z. Nedelkoski, D. Kepaptsoglou, L. Lari, T. Wen, R. A. Booth, S. D. Oberdick, P. L. Galindo, Q. M. Ramasse, R. F. L. Evans, S. Majetich and V. K. Lazarov, Sci. Rep., 2017, 7, 45997.

30 H. Sharifi Dehsari, V. Ksenofontov, A. Möller, G. Jakob and K. Asadi, J. Phys. Chem. C, 2018, 122, 28292-28301. 
31 Z. Nemati, J. Alonso, I. Rodrigo, R. Das, E. Garaio, J. Á. García, I. Orue, M.-H. Phan and H. Srikanth, J. Phys. Chem. C, 2018, 122, 2367-2381.

32 Ocean NanoTech LLC, Technical Specification of Iron Oxide Nanocrystals with Oleic Acid Coating, https://www.oceannanotech.com/media/wysiwyg/TSS/SOR_TSS.pdf, (accessed March 2021).

33 Ernst Ruska-Centre for Microscopy and Spectroscopy with Electrons, J. Large-Scale Res. Facil., 2016, 2, A77.

34 K. Koch, QSTEM: Quantitative TEM/STEM Simulations, 2018.

35 P. R. Willmott, D. Meister, S. J. Leake, M. Lange, A. Bergamaschi, M. Böge, M. Calvi, C. Cancellieri, N. Casati, A. Cervellino, Q. Chen, C. David, U. Flechsig, F. Gozzo, B. Henrich, S. Jäggi-Spielmann, B. Jakob, I. Kalichava, P. Karvinen, J. Krempasky, A. Lüdeke, R. Lüscher, S. Maag, C. Quitmann, M. L. Reinle-Schmitt, T. Schmidt, B. Schmitt, A. Streun, I. Vartiainen, M. Vitins, X. Wang and R. Wullschleger, J. Synchrotron Radiat., 2013, 20, 667-682.

36 P. Juhás, T. Davis, C. L. Farrow and S. J. L. Billinge, J. Appl. Crystallogr., 2013, 46, 560-566.

37 C. L. Farrow, P. Juhas, J. W. Liu, D. Bryndin, E. S. Božin, J. Bloch, T. Proffen and S. J. L. Billinge, J. Phys.: Condens. Matter, 2007, 19, 335219.

38 Jülich Centre for Neutron Science, GALAXI: Gallium anode low-angle x-ray instrument, J. Large-Scale Res. Facil., 2016, 2, A61, DOI: 10.17815/jlsrf-2-109.

39 A. Hammersley, J. Appl. Crystallogr., 2016, 49, 646-652.

40 R. Biehl, Jscatter, A Program For Evaluation And Analysis of Experimental Data, 2018.

41 J. S. Pedersen, D. Posselt and K. Mortensen, J. Appl. Crystallogr., 1990, 23, 321-333.

42 Hilgenberg $\mathrm{GmbH}$, Technical data sheet 0500, version 1-09/ 2003.

43 Heinz Maier-Leibnitz Zentrum, et al., J. Large-Scale Res. Facil., 2015, 1, A28.

44 A. V. Feoktystov, H. Frielinghaus, Z. Di, S. Jaksch, V. Pipich, M.-S. Appavou, E. Babcock, R. Hanslik, R. Engels, G. Kemmerling, H. Kleines, A. Ioffe, D. Richter and T. Brückel, J. Appl. Crystallogr., 2015, 48, 61-70.

45 V. Pipich, QtiKWS: user-friendly program for reduction, visualization, analysis and fit of $S A(N) S$ data, 2012.

46 A. Guinier, G. Fournet and K. L. Yudowitch, Small-angle scattering of X-rays, Wiley, New York, 1955.

47 K. Butter, A. Hoell, A. Wiedenmann, A. V. Petukhov and G.-J. Vroege, J. Appl. Crystallogr., 2004, 37, 847-856.

48 M. F. Hansen and S. Mørup, J. Magn. Magn. Mater., 1999, 203, 214-216.

49 D. Peddis, C. Cannas, A. Musinu and G. Piccaluga, J. Phys. Chem. C, 2008, 112, 5141-5147.

50 S. Bedanta, O. Petracic and W. Kleemann, Handbook of magnetic materials, Elsevier, 2015, vol. 23, pp. 1-83.

51 J. Landers, S. Salamon, H. Remmer, F. Ludwig and H. Wende, ACS Appl. Mater. Interfaces, 2018, 11, 3160-3168.

52 K. Pisane, S. Singh and M. Seehra, Appl. Phys. Lett., 2017, 110, 222409.
53 J. K. Vassiliou, V. Mehrotra, M. W. Russell, E. P. Giannelis, R. McMichael, R. Shull and R. F. Ziolo, J. Appl. Phys., 1993, 73, 5109-5116.

54 O. Petracic, X. Chen, S. Bedanta, W. Kleemann, S. Sahoo, S. Cardoso and P. Freitas, J. Magn. Magn. Mater., 2006, 300, 192-197.

55 M.-H. Phan, J. Alonso, H. Khurshid, P. Lampen-Kelley, S. Chandra, K. Stojak Repa, Z. Nemati, R. Das, Ó. Iglesias and H. Srikanth, Nanomaterials, 2016, 6, 221.

56 H. Khurshid, W. Li, S. Chandra, M.-H. Phan, G. C. Hadjipanayis, P. Mukherjee and H. Srikanth, Nanoscale, 2013, 5, 7942-7952.

57 S. K. Arora, R. Sofin, A. Nolan and I. V. Shvets, J. Magn. Magn. Mater., 2005, 286, 463-467.

58 J. B. Goodenough, Phys. Rev., 1955, 100, 564-573.

59 J. Kanamori, J. Phys. Chem. Solids, 1959, 10, 87-98.

60 W. Eerenstein, T. Palstra and T. Hibma, Thin Solid Films, 2001, 400, 90-94.

61 J. Park, K. An, Y. Hwang, J.-G. Park, H.-J. Noh, J.-Y. Kim, J.-H. Park, N.-M. Hwang and T. Hyeon, Nat. Mater., 2004, 3, 891-895.

62 K. P. McKenna, F. Hofer, D. Gilks, V. K. Lazarov, C. Chen, Z. Wang and Y. Ikuhara, Nat. Commun., 2014, 5, 5740.

63 P. Scherrer, Kolloidchemie Ein Lehrbuch, Springer, Berlin, Heidelberg, 1912, pp. 387-409.

64 U. Holzwarth and N. Gibson, Nat. Nanotechnol., 2011, 6, 534-534.

65 A. Monshi, M. R. Foroughi and M. R. Monshi, World J. Nano Sci. Eng., 2012, 2, 154-160.

66 B. D. Cullity and S. Stock, Elements of X-ray Diffraction, Prentice-Hall, 3rd edn, 2001.

67 G. Kugel, B. Hennion and C. Carabatos, Phys. Rev. B: Condens. Matter Mater. Phys., 1978, 18, 1317.

68 N. Guigue-Millot, N. Keller and P. Perriat, Phys. Rev. B: Condens. Matter Mater. Phys., 2001, 64, 012402.

69 G. Goya, T. Berquo, F. Fonseca and M. Morales, J. Appl. Phys., 2003, 94, 3520-3528.

70 M. Coduri, P. Masala, L. D. Bianco, F. Spizzo, D. Ceresoli, C. Castellano, S. Cappelli, C. Oliva, S. Checchia, M. Allieta, D.-V. Szabo, S. Schlabach, M. Hagelstein, C. Ferrero and M. Scavini, Nanomaterials, 2020, 10, 867.

71 R. Grau-Crespo, A. Y. Al-Baitai, I. Saadoune and N. H. de Leeuw, J. Phys.: Condens. Matter, 2010, 22, 255401.

72 J.-E. Jørgensen, L. Mosegaard, L. E. Thomsen, T. R. Jensen and J. C. Hanson, J. Solid State Chem., 2007, 180, 180185.

73 T. Proffen, S. J. L. Billinge, T. Egami and D. Louca, Z. Kristallogr. - Cryst. Mater., 2003, 218, 1203.

74 K. Kelm and W. Mader, Z. Naturforsch., B: J. Chem. Sci., 2006, 61, 665-671.

75 C. Greaves, J. Solid State Chem., 1983, 49, 325-333.

76 A. Cervellino, R. Frison, G. Cernuto, A. Guagliardi and N. Masciocchi, J. Appl. Crystallogr., 2014, 47, 1755-1761.

77 A. G. Roca, J. F. Marco, M. D. P. Morales and C. J. Serna, J. Phys. Chem. C, 2007, 111, 18577-18584. 
78 J. Landers, F. Stromberg, M. Darbandi, C. Schöppner, W. Keune and H. Wende, J. Phys.: Condens. Matter, 2014, 27, 026002.

79 P. Sidhu, R. Gilkes and A. Posner, J. Inorg. Nucl. Chem., 1977, 39, 1953-1958.

80 M. Morales, C. Serna, F. Bødker and S. Mørup, J. Phys.: Condens. Matter, 1997, 9, 5461.

81 D. Li, W. Y. Teoh, C. Selomulya, R. C. Woodward, P. Munroe and R. Amal, J. Mater. Chem., 2007, 17, 48764884 .

82 F. Wiekhorst, E. Shevchenko, H. Weller and J. Kötzler, Phys. Rev. B: Condens. Matter Mater. Phys., 2003, 67, 224416.

83 S. J. Kemp, R. M. Ferguson, A. P. Khandhar and K. M. Krishnan, $R S C A d v .$, 2016, 6, 77452-77464.

84 P. Tartaj, T. González-Carreño and C. J. Serna, J. Phys. Chem. B, 2003, 107, 20-24.
85 P. Guardia, B. Batlle-Brugal, A. Roca, O. Iglesias, M. Morales, C. Serna, A. Labarta and X. Batlle, J. Magn. Magn. Mater., 2007, 316, e756-e759.

86 A. Wiedenmann, J. Appl. Crystallogr., 2000, 33, 428-432.

87 A. Michels, J. Phys.: Condens. Matter, 2014, 26, 383201.

88 S. Mühlbauer, D. Honecker, É. A. Périgo, F. Bergner, S. Disch, A. Heinemann, S. Erokhin, D. Berkov, C. Leighton, M. R. Eskildsen and A. Michels, Rev. Mod. Phys., 2019, 91, 015004.

89 M. Avdeev, D. Bica, L. Vekas, V. Aksenov, A. Feoktystov, O. Marinica, L. Rosta, V. Garamus and R. Willumeit, J. Colloid Interface Sci., 2009, 334, 37-41.

90 M. V. Avdeev, J. Appl. Crystallogr., 2007, 40, 56-70.

91 D. Zákutná, D. Niznansky, L. Barnsley, A. Feoktystov, D. Honecker and S. Disch, Phys. Rev. X, 2020, 10, 031019.

92 S. Arora, R. Sofin and I. Shvets, Phys. Rev. B: Condens. Matter Mater. Phys., 2005, 72, 134404. 